Jost B Jonas, Florian Rensch

Department of Ophthalmology, Medical Faculty Mannheim of the Ruprecht-Karls-University Heidelberg, Germany

Correspondence to: Dr J Jonas, UniversitätsAugenklinik, Theodor-Kutzer-Ufer 1-3, 68167 Mannheim, Germany; Jost.Jonas@ma.augen.uni-heidelberg.de

\section{doi: 10.1136/bjo.2007.116558}

Accepted 13 February 2007

Competing interests: None.

\section{References}

1 Greenberg PB, Martidis A, Rogers AH, et al. Intravitreal triamcinolone acetonide for macular oedema due to central retinal vein occlusion. Br J Ophthalmol 2002;86:247-8.

2 Ip MS, Kumar KS. Intravitreous triamcinolone acetonide as treatment for macular edema from central retinal vein occlusion. Arch Ophthalmol 2002; 120:1217-19.

3 Jonas JB, Kreissig I, Degenring RF. Intravitreal triamcinolone acetonide as treatment of macular edema in central retinal vein occlusion. Graef Arch Clin Exp Ophthalmol 2002;240:782-3.

4 Park CH, Jaffe GJ, Fekrat S. Intravitreal triamcinolone acetonide in eyes with cystoid macular edema associated with central retinal vein occlusion. Am J Ophthalmol 2003;136:419-25.

5 Cekic O, Chang S, Tseng JJ, et al. Intravitreal triamcinolone injection for treatment of macular edema secondary to branch retinal vein occlusion. Retina 2005;25:851-5.

\section{Non-penetrating deep sclerectomy for glaucoma surgery using the femtosecond laser: a laboratory model}

Non-penetrating deep sclerectomy (NPDS) is a non-perforating filtration procedure used for the surgical treatment of medically uncontrolled open angle glaucoma. This procedure was developed in an attempt to avoid many of the postoperative complications of trabeculectomy. ${ }^{1}$ The major advantage of NPDS is that it precludes the sudden hypotony that occurs after trabeculectomy by creating progressive filtration of aqueous humour from the anterior chamber to the subconjunctival space, without perforating the eye. ${ }^{2}$ Preservation of the thin trabeculo-Descemet's membrane, however, is technically challenging, particularly before the surgeon gains experience with this procedure.

Previous studies investigated the ability to use the femtosecond laser for photodisruption in the human sclera, ${ }^{3}$ and concluded that complete subsurface photodisruption can be accomplished in human sclera in vitro.

Toyran et al. ${ }^{5}$ in 2005 published their in-vitro study that tested the feasibility of using femtosecond laser pulses to fistulise the human trabecular meshwork and concluded that, with appropriate exposure time and pulse energy, femtosecond photodisruption can be employed to create partial and full thickness ablation in the human trabecular meshwork without damaging the surrounding tissues.

In the study described here, we used femtosecond laser technology to perform subsurface photodisruption in the opaque sclera for NPDS surgery in a laboratory model.

\section{Materials and methods}

Eight NPDS operations were performed in five cadaver eyes for this study.
The donor eye was placed in an eye holder with a suction ring to immobilize it.

Conjunctiva and Tenon's capsule were reflected. A $200 \mu \mathrm{m}$ thick, limbal-based, $7 \mathrm{~mm}$ diameter round scleral flap was performed using the femtosecond laser (IntraLase Corp Irvine, California, USA). The IntraLase used a raster spot pattern for creating 1-2 micron bubbles with bed energy of $2.6 \mu \mathrm{J}$. The spot separation was $6 / 7 \mu \mathrm{m}$ and the firing rate was $60 \mathrm{kHz}$. The hinge was placed $1-2 \mathrm{~mm}$ into clear cornea (fig 1). The scleral flap separation was started on the corneal side, using a Sibel spatula, and was continued in the same plane into the sclera using corneal dissectors. The flap was lifted and a second circular flap of 400 microns depth, $5 \mathrm{~mm}$ diameter, was performed, dissected (fig 2A) and amputated (fig $2 \mathrm{~B}$ ), leaving a thin layer of deep sclera over the choroid posteriorly. Anteriorly, the dissection was into Schlemm's canal, which was unroofed. The corneal stroma was excised to the level of Descemet's membrane and aqueous humour began percolating through the remaining thin trabeculoDescemet's membrane. Methylene blue was injected to the anterior chamber, using a 25 gauge needle, in order to demonstrate aqueous humour percolation (fig 2B). The superficial scleral flap could then be repositioned and sutured with two single 10-0 nylon sutures.

\section{Results}

The creation of a scleral flap was achieved using an IntraLase without damage to overlying tissue in all eight NPDS operations. A thinned sclera was observed underneath the flaps with percolating aqueous humour (fig 1).

One case had an intraoperative perforation of the sclera with choroid prolapse after performing the second scleral flap with the femtosecond laser.

\section{Discussion}

Despite the advantages of decreased postoperative complications when compared with trabeculectomy in primary open angle glaucoma surgery, deep sclerectomy has not gained wide acceptance partly because of the difficulty of the technique, with a long learning curve, and as a result of studies that indicate that NPDS is less effective than trabeculectomy for

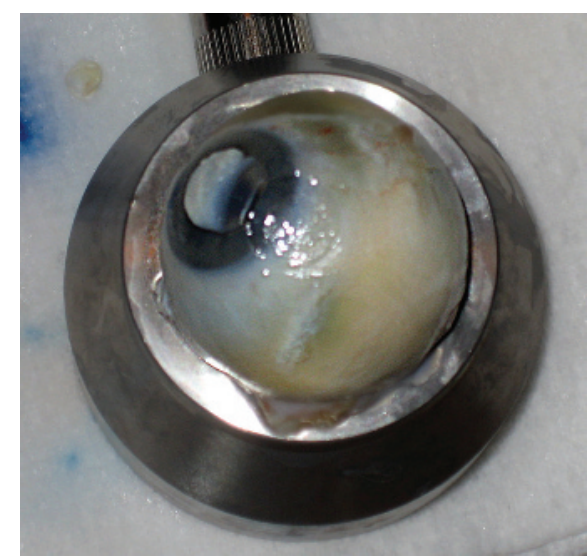

Figure 1 Photograph of the first limbal-based, $200 \mu \mathrm{m}$ thick, $7 \mathrm{~mm}$ diameter circular scleral flap performed on cadaver eye using the femtosecond laser. Flap was dissected using a Siebel spatula and cornea dissectors and lifted on the cornea.
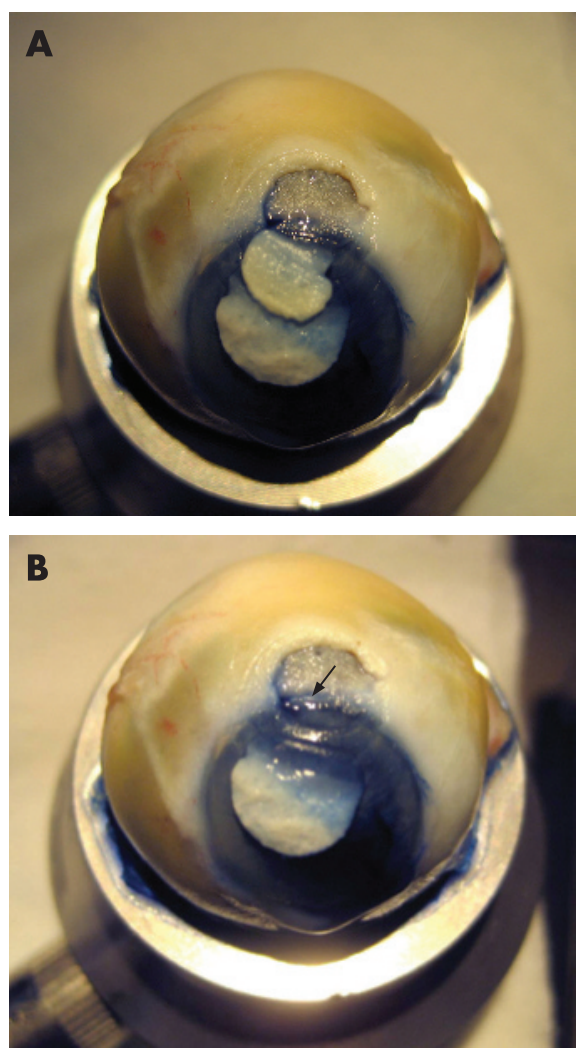

Figure 2 Photograph of the second, $400 \mu \mathrm{m}$ thick, $5 \mathrm{~mm}$ diameter circular scleral flap performed on cadaver eye using the femtosecond laser. The flap was dissected and the two flaps were lifted on the cornea (A). Methylene blue was injected into the anterior chamber. The second flap was amputated, the anterior trabeculum and Descemet's membrane were exposed, and methylene blue percolated through the remaining membrane (arrow) (B).

intraocular pressure reduction on a medium and long-term basis. ${ }^{6}$ When intrascleral collagen implants are used, however, deep sclerectomy yields improved results according to some authors. ${ }^{7}$ Our preliminary study on cadaver eyes suggests that high precision, subsurface scleral photodisruption can be achieved for NPDS surgery, using the femtosecond laser. This could simplify and facilitate this surgical procedure.

The major known intraoperative complication of this surgery is perforation of the thin trabeculo-Descemet's membrane during the deep sclerectomy dissection. ${ }^{8}$. No case of intraoperative perforation of the trabeculoDescemet's membrane was observed in all eight surgeries performed by us using the femtosecond laser.

Lifting of the scleral portion of the flap was harder than the corneal one. We thus had to use higher bed energies and a sharper corneal dissector to complete the scleral dissection at the same plane as the corneal.

One case of scleral perforation and choroidal prolapse occurred in our series. This could be attributed to a deeper dissection plane in the sclera made by the surgeon using the cornea dissectors during dissection of the first flap (which was thicker than planned). Therefore, when the second circular flap of 400 microns depth, $5 \mathrm{~mm}$ diameter was performed, it perforated the sclera. 
One limitation of this study is the nonhealing nature of this laboratory model, which does not allow an assessment of the long-term stability of the wound and efficacy of the procedure in terms of reducing intraocular pressure.

In conclusion, we have shown here that scleral dissection for NPDS surgery can be accurately achieved using a femtosecond laser. Further investigation on an animal model with clinical correlations is needed.

\section{Acknowledgements}

The authors would like to thank Intralase Corp. for support of this study.

\section{Irit Bahar, Igor Kaiserman, Graham E Trope, David Rootman}

Department of Ophthalmology, Toronto Western Hospital, University of Toronto, Toronto, Ontario

Canada

Correspondence to: Dr Irit Bahar, Department of Ophthalmology, Toronto Western Hospital, 399 Bathurst Street, Ontario, Canada M5T 2S8; iritbahar@ yahoo.com

doi: 10.1136/bjo.2007.116632

Accepted 20 February 2007

Competing interests: None declared.

\section{References}

1 Watson PG, Jakeman C, Ozturk M, et al. The complications of trabeculectomy (a 20-year followup). Eye 1990;4:425-38

2 Chiou AGY, Mermoud A, Hédiguer SEA, et al. Ultrasound biomicroscopy of eyes undergoing deep sclerectomy with collagen implant. Br J Ophthalmol 1996;80:541-4.

3 Sacks ZS, Kurtz RM, Juhasz T, et al. High precision subsurface photodisruption in human sclera. J Biomed Opt 2002;7:442-50.

4 Sacks ZS, Kurtz RM, Juhasz T, et al. Subsurface photodisruption in human sclera: wavelength dependence. Ophthalmic Surg Lasers Imaging 2003;34:104-13

5 Toyran S, Liu Y, Singha S, et al. Femtosecond laser photodisruption of human trabecular meshwork: an in vitro study. Exp Eye Res 2005;81:298-305.

6 Jonescu-Cuypers C, Jacobi P, Konen W, et al. Primary viscocanalostomy versus trabeculectomy in white patients with open angle glaucoma: a randomized clinical trial. Ophthalmology 2001;108:254-8.

7 Mermoud A, Schnyder CC, Sickenberg M, et al. Comparison of deep sclerectomy with collagen implant and trabeculectomy in open-angle glaucoma. J Cataract Refract Surg 1999;25:323-31.

8 Lachkar Y, Hamard P. Nonpenetrating filtering surgery. Curr Opin Ophthalmol 2002;13:110-15.

9 Yamin M, Quentin CD. Results and complications after deep sclerectomy. Ophthalmologe 2002;99:171-5.

\section{Pulmonary tuberculosis presenting with central retinal vein occlusion}

Tuberculosis remains a major international public health concern, with the global incidence rate increasing approximately $0.4 \%$ per year, but much faster in sub-Saharan Africa and Eastern Europe; ${ }^{1}$ the effect that HIV has on incidence is also highly significant. ${ }^{2}$ Within the UK the incidence of tuberculosis varies considerably between regions and ethnic groups, with rates increasing significantly among recent immigrants. ${ }^{3}$

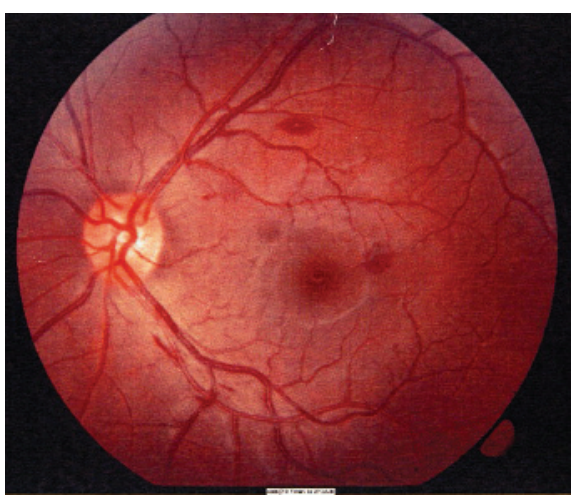

Learning points

- Any cause of central retinal vein thrombosis should be followed up and a medical cause sought, a chest X-ray being an appropriate baseline investigation.

- Early recognition of this association is vital to limit morbidity.

- Tuberculosis can present in a variety of ways and should be thought of in the differential diagnosis especially in recent immigrants to the United Kingdom.

Figure 1 Image of left retina showing central retinal vein occlusion and retinal haemorrhage.

This case describes a patient who presented with central retinal vein occlusion (CRVO) who was subsequently diagnosed with tuberculosis.

\section{Case report}

A 28-year-old Asian woman presented with transient obscuration of her vision. She had no other eye symptoms, central nervous system or systemic symptoms. She had no history of fevers or night sweats. There were no respiratory symptoms. There was no past medical history. She had lived in the United Kingdom for 18 months and had an apparently normal chest X-ray in her home country before leaving to come to the United Kingdom. She was a non-smoker with no significant family history of illness.

On examination she was thin but she did not report significant weight loss. There were small lymph nodes in her left cervical chain. Fundoscopy revealed CRVO (see fig 1). Initial investigations showed normal urea and electrolytes, white cell count of $6.410^{9}$ cells/l, haemoglobin $12.2 \mathrm{~g} / \mathrm{dl}$, platelets 318 , normal clotting screen, C-reactive protein $3.6 \mathrm{mg} / \mathrm{l}$, erythrocyte sedimentation rate $38 \mathrm{~mm} /$ hour total protein was elevated at $87 \mathrm{~g} / \mathrm{l}$, plasma viscosity was elevated at $1.95 \mathrm{pa} / \mathrm{s}$, IgG was $20.51 \mathrm{~g} / \mathrm{l}$. She had negative results for the following: rheumatoid factor, anti-nuclear antibody, lupus anti-coagulant, IgG and IgM phospholipid antibody, neutrophil cytoplasmic antibody (both anti-proteinase cytoplasmic

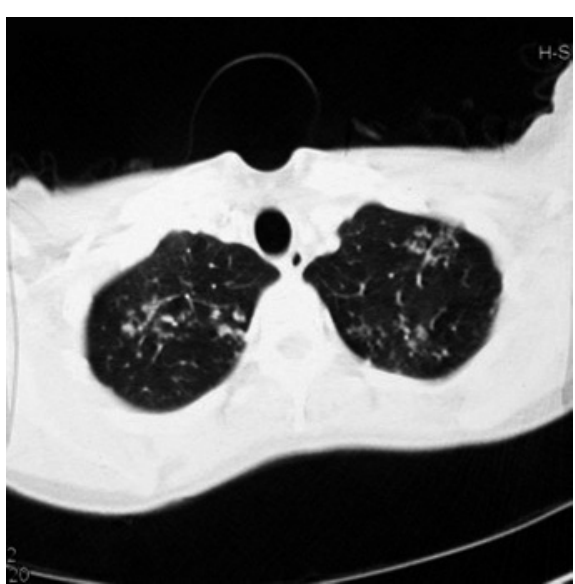

Figure 2 High resolution of computed tomography scan of thorax showing 'tree in bud' opacities in apical segments of upper lobes. and myeloperoxidase perinuclear antineutrophil cytoplasmic antibody), and cryoglobulins. Complement C3 and C4 levels were normal.

Chest X-ray showed very faint inflammatory shadowing in the left apex and a bulge in the contour of the right hilum possibly representing lymphadenopathy. She had a normal magnetic resonance imaging brain scan. A computed tomography scan of the abdomen and thorax (fig 2) was performed that showed significant lymphadenopathy in the cervical and supraclavicar region as well the right hilum, subcarinal and paratracheal regions. The subcarinal lymph nodes were heavily calcified. There were 'tree in bud' opacities in the apical segments of the upper lobes, suggestive of active pulmonary tuberculosis.

She had a bronchoscopy as she was unable to expectorate sputum. Bronchoscopic washings of the upper lobes showed scanty acid-fast bacilli. Fully sensitive Mycobacterium tuberculosis grew from the culture. This confirmed the diagnosis, central retinal vein thrombosis associated with active tuberculosis. She has made a full recovery on quadruple antituberculous therapy, with resolution of her eye symptoms.

\section{Comment}

In this case symptoms were caused by nonischaemic CRVO, which on its own does not lead to blindness and carries a good prognosis. Ischaemic CRVO, however, is the second commonest sight-threatening vascular disorder; the pathogenesis remains unclear. ${ }^{4}$ The commonest associations are hypertension, diabetes mellitus, bleeding or clotting disorders, autoimmune conditions, glaucoma, head trauma or excess alcohol consumption. Many other aetiologies have, however, been reported including secondary to excess liquorice ingestion, renal cell carcinoma, multiple myeloma, herpesvirus 6, drugs (including sildenafil and rofecoxib) and as a result of paraneoplatic phenomenon associated with lung cancer.

A retrospective study of 103 cases of CRVO in young adults without diabetes who were followed for at least six months showed the majority $(64 \%)$ to be men. Although vision was good approximately a third had vision of 20/ 200 or worse. ${ }^{5}$ In a series of 17 young UK patients with CRVO, ocular involvement was characteristically unilateral, with moderate degrees of retinal haemorrhage, little retinal ischaemia, and a tendency to optic disc swelling. Visual prognosis was found to be good and follow-up showed that most patients had good general health and no involvement of the other eye. Interestingly, an inflammatory aetiology or underlying vascular disease was not found in most patients. 